\title{
Cyclooxygenase-2 inhibitor celecoxib attenuates joint contracture following immobilization in rat knees
}

\author{
Junya Ozawa ${ }^{1 *}$, Akinori Kaneguchi², Ryo Tanaka' ${ }^{1}$ Nobuhiro Kito ${ }^{1}$ and Hideki Moriyama ${ }^{3}$
}

\begin{abstract}
Background: The aim of this study is to clarify the following two points: First, whether a cyclooxygenase-2 mediated pathway is involved in the formation of immobilization-induced joint contracture and, second, the effectiveness of oral administration of non-steroidal anti-inflammatory drug celecoxib (CBX) for the prevention of myogenic and arthrogenic contracture following immobilization in a rat model.
\end{abstract}

Methods: Thirty male rats were randomly divided into three groups: immobilization (Im), Im + CBX, and control ( $n=10$ each). External fixation immobilized the right knee joint of $\mathrm{Im}$ and $\mathrm{Im}+\mathrm{CBX}$ groups in flexion for 3 weeks. $50 \mathrm{mg} / \mathrm{kg}$ of CBX was administrated daily to the $\mathrm{Im}+\mathrm{CBX}$ group during this period. The passive range of motion (ROM) of knee joints was measured before and after transection of knee flexor muscles and myogenic and arthrogenic ROM restrictions were calculated. The semitendinosus muscles and knee joints were investigated histologically to elucidate factors responsible for contracture.

Results: Myogenic ROM restrictions were exhibited both in Im and Im + CBX groups ( $44 \pm 5$ and $36 \pm 8^{\circ}$, respectively), but restrictions significantly decreased in the $\mathrm{Im}+\mathrm{CBX}$ group compared to the Im group. Significant reductions of the muscle length ratios (Rt/Lt) and sarcomere number ratios (Rt/Lt) in knee flexor semitendinosus muscle, which are responsible for myogenic contracture, were also seen both in Im group (92 \pm 5 and $92 \pm 4 \%$, respectively) and Im + CBX group (97 \pm 3 and $97 \pm 3 \%$, respectively), but were inhibited by CBX administration $(P<0.05)$. Im and Im + CBX groups exhibited arthrogenic ROM restrictions with no significant differences ( $82 \pm 3$ and $83 \pm 5^{\circ}$, respectively). Posterior synovial length shortening and pathological changes (hemorrhage in joint cavities and capsule edema) in the knee joints were comparable between Im and Im + CBX groups.

Conclusions: Oral administration of celecoxib partially reduced myogenic ROM restriction concomitantly with knee flexor muscle shortening following immobilization. These results imply that inflammation and nociception are involved in myogenic contracture formation independently of joint immobilization, and that CBX is effective in preventing joint contracture following immobilization in rats.

Keywords: Celecoxib, Joint contracture, Immobilization, Knee, Muscle

\footnotetext{
* Correspondence: j-ozawa@hs.hirokoku-u.ac.jp

${ }^{1}$ Department of Rehabilitation, Faculty of Rehabilitation, Hiroshima

International University, Kurose- Gakuendai 555-36, Higashi-Hiroshima,

Hiroshima 739-2695, Japan

Full list of author information is available at the end of the article
}

(c) The Author(s). 2016 Open Access This article is distributed under the terms of the Creative Commons Attribution 4.0 International License (http://creativecommons.org/licenses/by/4.0/), which permits unrestricted use, distribution, and reproduction in any medium, provided you give appropriate credit to the original author(s) and the source, provide a link to the Creative Commons license, and indicate if changes were made. The Creative Commons Public Domain Dedication waiver (http://creativecommons.org/publicdomain/zero/1.0/) applies to the data made available in this article, unless otherwise stated. 


\section{Background}

The development of joint contractures is induced by a variety of events such as immobilization [1-6], joint surgery or trauma [7], muscle weakness, and neurological dysfunction such as paralysis and spasticity $[1,8]$. These events accompany joint inactivity and suggest that joint immobilization is essentially a trigger for contracture formation. If movement of the affected joint is permitted, passive movements (e.g., stretching, continuous passive motion, and splinting) are the most common interventions for the treatment and prevention of joint contractures [1]. However, the efficacy of passive movement is still controversial. A systematic review revealed that dynamic splints, which provide prolonged passive stretching, is a safe and effective treatment for contracture in humans and animals [8]. On the other hand, Prabhu et al. [9] reviewed that it is not clear whether passive movements are effective for the treatment and prevention of contractures. Katalinic et al. [10] also concluded that stretching does not have an important effect on joint mobility. These results imply the need for the development of new alternative methods for the treatment of joint contractures.

In a recent study, we found that contracture is generated in arthritic knee joints with only a slight, transient reduction in active joint range of motion (ROM) during locomotion [11, 12]. This result indicates that joint contracture is not necessarily associated with joint immobilization. Interestingly, joint immobilization leads to not only contracture, but also joint inflammatory conditions characterized by synovitis [13] and an upregulation of inflammation-related genes [14]. In addition, arthritis-induced contracture is partially prevented and improved by anti-inflammatory drugs [15-17]. As such, anti-inflammatory treatment may prevent immobilizationinduced contracture as well as arthritis-induced contracture. However, investigation into the effects of anti-inflammatory drugs on immobilization-induced joint contracture is very limited. Michelsson et al. [18] reported that administration of corticosteroid methylprednisolone has a preventive effect on joint stiffening, but only in the beginning of the immobilization period ( $<5-6$ weeks). After this, ROM restriction proceeded despite remobilization of the rabbit knees. It was also shown that the administration of ibuprofen, a non-steroidal anti-inflammatory drug (NSAID), does not suppress the formation of joint stiffening in immobilized rabbit knees [19]. However, ROM analysis was only carried out semiquantitatively, and more importantly, the report did not distinguish between the contributions of muscular and articular factors on contracture formation; the factors responsible for joint contracture are divided into mainly muscular and periarticular structures $[4,20]$. Intra-articular injection of high molecular weight hyaluronan, which modulates inflammatory response, could prevent fibrotic changes in immobilized joint capsules in rats [21] and rabbits [22]. However, they did not evaluate myogenic ROM restriction and focused not on muscular but on articular factors in the contribution of contracture. Thus, the effects of antiinflammatory drugs on myogenic and arthrogenic contracture formation have not been yet concluded.

In this study, we selected celecoxib (SC-58635; 4-[5-(4methylphenyl)-3-(trifluoromethyl)-1H-pyrazol-1-yl] benzenesulfonamide), a selective cyclooxygenase (COX)-2 inhibitor, as the anti-inflammatory drug. Celecoxib has been widely used to treat pain and inflammation via suppression of prostaglandin $\mathrm{E}_{2}$ synthase [23]. The aim of this study is to clarify the following two points: First, whether a COX-2 mediated pathway is involved in the formation of immobilization-induced joint contracture and, second, the effectiveness of NSAID celecoxib for the prevention of myogenic and arthrogenic contracture following immobilization in a rat model.

\section{Methods \\ Animals}

Eight-week-old male Wistar rats (30 animals, $199 \pm 3$ g, Japan SLC, Shizuoka, Japan) were used in this study. The animals were housed in standard cages, given access to food and water ad libitum, and maintained in a thermoneutral environment $\left(22-25{ }^{\circ} \mathrm{C}\right)$ with a 12 -h light-dark cycle.

\section{Joint immobilization}

Joint immobilization was conducted using the external fixation method, as described previously [24]. Under anesthesia with an intraperitoneal injection of sodium pentobarbital $(0.5 \mathrm{~mL} / \mathrm{kg})$, Kirschner wires were screwed into the femur and tibia of the rat's right hindlimbs and subsequently fixed by wire and resin at $140{ }^{\circ}$ knee flexion for 3 weeks (Fig. 1). Joint immobilized rats were divided into two groups: immobilization (Im) $(n=10)$ and immobilization with celecoxib administration ( $\mathrm{Im}+$ CBX) $(n=10)$. Celecoxib (50 mg/kg orally) was administrated daily to the Im + CBX group during the 3 week immobilized period. Age-matched untreated animals were used as the control group $(n=10)$.

\section{ROM measurement}

At the end of the intervention period, passive knee joint ROM was measured as described previously [12]. Rats were anesthetized with sodium pentobarbital $(0.5 \mathrm{~mL} / \mathrm{kg})$ with supplemental diethyl ether inhalation to inhibit muscle contraction completely, if necessary. After removing an external fixator and exfoliating of skin of the hindlimb, color markers were placed corresponding to the greater trochanter, knee lateral joint space, and lateral malleolus. The femur was fixed at approximately $90^{\circ}$ hip 


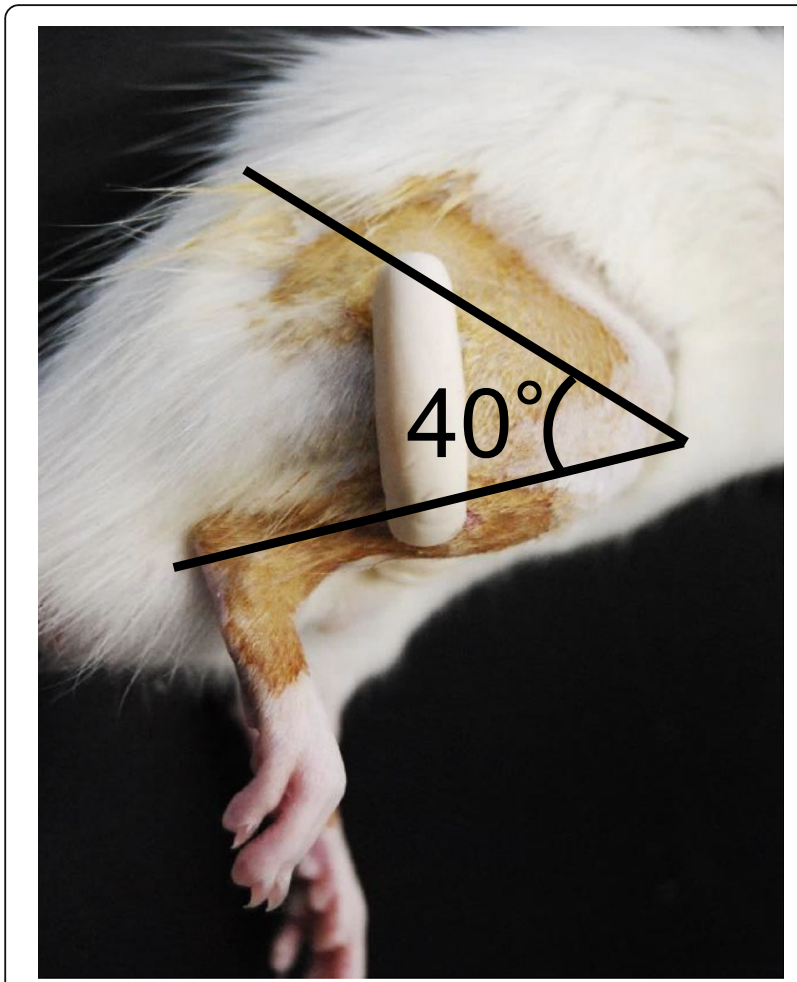

Fig. 1 Photograph of joint immobilization. Knee joint is immobilized by an external fixator at approximately $140^{\circ}$ flexion (the angle between the femur and the tibia is $40^{\circ}$ )

flexion, and 14.6 N.mm extension moments were applied to the knee joint. Knee joint ROM was measured using a 3D motion analysis system KinemaTracer (KISSEI COMTEC, Nagano, Japan) and three video cameras (Tokina, Tokyo, Japan). The markers in the filmed image were traced. The KinemaTracer software used to analyze the coordinates was calibrated by recording a cube of known size. The angles between the axes of the femur (the greater trochanter to the knee lateral joint space) and fibula (the knee lateral joint space to the lateral malleolus) were measured as the knee extension ROM $(\mathrm{ROM}+\mathrm{m})$. After the $\mathrm{ROM}+\mathrm{m}$ measurement, rats were sacrificed by exsanguination under inhalation of diethyl ether. The knee flexor muscles, except for the semitendinosus muscles, were completely transected at the mid-belly portion. As for the semitendinosus muscle, the ischium tuberosity attached to the tendon was removed from the hip bone. After excluding myogenic factors in flexion contracture, knee extension ROM (ROM-m) was measured again as detailed above. All measurements were performed on both sides of the knee in all groups.

Myogenic ROM restriction was defined as contracture due to knee flexor muscle components, and arthrogenic ROM restriction was defined as contracture due to articular components such as bone, cartilage, synovium, subsynovium, capsule, and ligaments. These ROMs were calculated using the methods of Moriyama et al. [25]. The formulas are as follows:

$$
\begin{aligned}
\text { Myogenic restriction }= & \mathrm{ROM}-\mathrm{m}-\mathrm{ROM}+\mathrm{m} \\
& (\text { within each treatment group }) \\
\text { Arthrogenic restriction }=\mathrm{ROM}-\mathrm{m} \text { of control group }-\mathrm{ROM}-\mathrm{m} & \text { of each treatment group. }
\end{aligned}
$$

\section{Tissue preparation}

After the ROM measurement, the semitendinosus muscles attached to the bones (ischium and tibia), were immediately removed from both hindlimbs. The distal end of the muscles were fixed and the proximal end was connected to $16 \mathrm{~g}$ of weight to stretch them with a uniform force using a pulley in $0.1 \mathrm{M}$ phosphatebuffered $4 \%$ paraformaldehyde $(\mathrm{pH} \mathrm{7.4)}$ for $4 \mathrm{~h}$ at room temperature. The weight was determined beforehand as the same force required for the knee joint extension at $140^{\circ}$, which is approximately the maximal extension ROM in a normal joint. After fixation, muscle length was measured using manually operated calipers (Mitutoyo Instruments, Yokohama, Tokyo) and the midbelly portion of muscles was dissected and embedded in paraffin. Longitudinal sections $(4-\mu \mathrm{m})$ were cut and stained with hematoxylin and eosin (HE) for microscopic observation.

Joint tissues were also dissected and immersion-fixed in the same fixative for 2 days at $4{ }^{\circ} \mathrm{C}$ to investigate knee joint pathology. During fixation, the knee joint angle was kept at $90^{\circ}$ flexion. Joint samples were decalcified in $17.7 \%$ ethylendiamintetraacetic acid ( $\mathrm{pH}$ 7.2, OSTEOSOFT, Merck Millipore, Darmstadt, Germany) for 4 weeks at room temperature, cut into two pieces on the sagittal plane, and embedded in paraffin. The sections $(4-\mu \mathrm{m})$ were cut at the central level of the medial condyle and stained with HE and aldehyde-fuchsin-Masson Goldner (AFMG) staining for histological study.

\section{Histological and histomorphometrical analysis The semitendinosus muscles}

Three portions from each HE section were photographed at a magnification of $20 \mathrm{x}$ with a digital camera mounted on a light microscope to visualize sarcomeres. Ten muscle fibers from each sample were randomly selected and the myofibril lengths were measured using Image J 1.48 software (National Institutes of Health, Bethesda, MD, USA). The number of serially arranged sarcomeres, including the length-measured myofibrils was counted and the average sarcomere lengths were calculated. In addition, the number of serially arranged sarcomeres, including the whole muscle, was calculated by dividing the muscle length by sarcomere length and expressing it as a total sarcomere number. 


\section{Knee joint hemorrhage score}

Intra-articular hemorrhages were detected through microscopic observation in the knee joints in both Im and Im + CBX groups. Two observers unaware of the source of the samples evaluated joint hemorrhage severity. Severity of the hemorrhage was graded from 0 to 4 depending on the amount of infiltrated blood-derived cells: $0=$ none, $1=$ slight, $2=$ moderate, $3=$ severe, and $4=$ more severe. The antero-superior, antero-inferior, postero-superior, and postero-inferior portions of joint cavities were graded separately. The sum of these individual scores averaged for the two observers was used as the representative score for each portions.

\section{Joint capsule length}

The length of the postero-superior and postero-inferior synovial membrane were measured using the image analysis software ImageJ according to the methods described by Ando et al. [26]. The measurements were summed to provide a total synovial membrane length. Shortened posterior synovial length is thought to be responsible for knee joint contracture in a rat immobilization and spinal cord injury model $[25,27,28]$.

\section{Statistical analysis}

All data were expressed as mean \pm standard deviation (SD). Statistical analysis was performed using Dr. SPSS II for Windows (SPSS Japan Inc, Tokyo, Japan). For all data except for the hemorrhage score, one-way analysis of variance (ANOVA) and Tukey's post-hoc test were applied. For the hemorrhage score, a nonparametric Kruskal-Wallis test was applied, followed by a Mann-Whitney test with the Bonferroni adjustment. A $P$-value of $<0.05$ was considered statistically significant.

\section{Results}

\section{Range of motion}

Knee extension ROMs before myotomy $(\mathrm{ROM}+\mathrm{m})$ and after myotomy (ROM-m) of knee flexor muscles on both sides are shown in Fig. 2. The $\mathrm{ROM}+\mathrm{m}$ on the experimental (Rt) side were $145 \pm 5,88 \pm 4$, and $102 \pm 9^{\circ}$ in the control, Im, and Im $+\mathrm{CBX}$ groups, respectively (Fig. 2a). The $\mathrm{ROM}+\mathrm{m}$ on the experimental side decreased in both Im and Im + CBX groups compared to each contralateral (Lt) side and the control group $\left(P<0.0005, \mathrm{~F}=240.9, \eta_{\mathrm{P}}^{2}=0.95\right.$, one-way ANOVA; all $P<0.05$, Tukey's post hoc test). In comparing the two immobilized groups, $\mathrm{ROM}+\mathrm{m}$ of the $\mathrm{Im}+\mathrm{CBX}$ group was larger than the Im group $(P<0.05$, Tukey's post hoc test). The ROM-m on the experimental side were $160 \pm 7,132 \pm 5$, and $137 \pm 9^{\circ}$ in the control, Im, and Im + CBX groups, respectively (Fig. 2b). The ROM-m on the experimental side also decreased in both Im

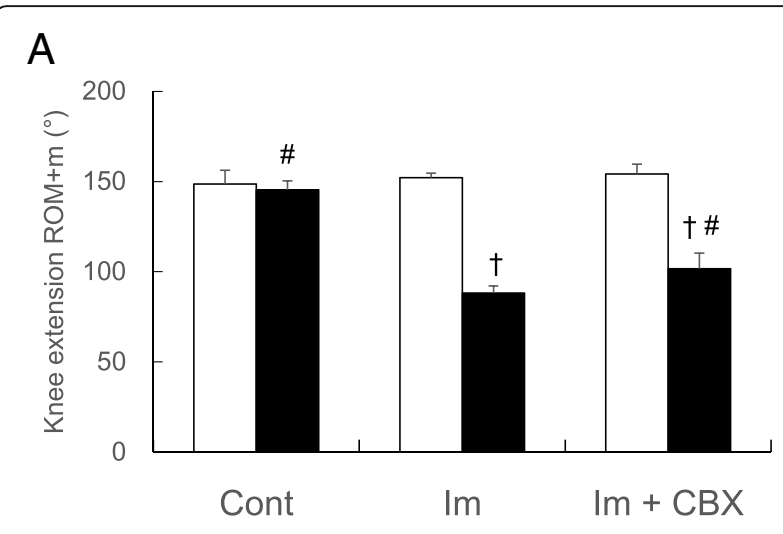

B

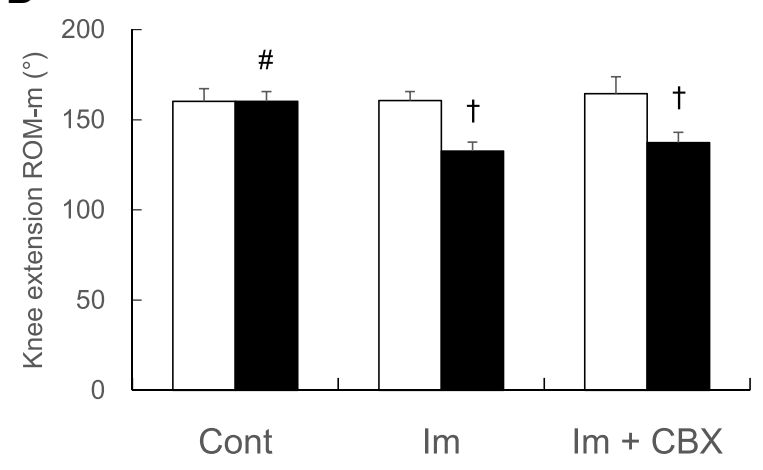

Fig. 2 Histograms showing changes in passive knee extension ROMs. a ROM before myotomy of knee flexor muscles (ROM+m). Joint immobilization induced $\mathrm{ROM}+\mathrm{m}$ restrictions in both $\mathrm{Im}$ and $\mathrm{Im}+\mathrm{CBX}$ groups. Celecoxib partially prevented the $\mathrm{ROM}+\mathrm{m}$ restriction induced by joint immobilization. b ROM after myotomy of knee flexor muscles (ROM-m). Joint immobilization induced ROM-m restrictions in both Im and Im + CBX groups. Celecoxib did not change ROM-m restriction induced by joint immobilization. (Experimental side (Rt), $\mathbf{\square}$; Contralateral side (Lt), $\square) .{ }^{\dagger} P<0.05$ vs. contralateral side of the same group; ${ }^{\sharp} P<0.05$ vs. ipsilateral side of Im group. Data are expressed as means \pm SD $(n=10)$

and $\mathrm{Im}+\mathrm{CBX}$ groups compared to each contralateral side and the control group $\left(P<0.0005, \mathrm{~F}=41.7, \eta_{\mathrm{P}}^{2}=\right.$ 0.75, one-way ANOVA; all $P<0.05$, Tukey's post hoc test). Contrary to the result in $\mathrm{ROM}+\mathrm{m}$, there was no difference in ROM-m between the Im and Im + CBX groups $(P=0.38$, Tukey's post hoc test).

Myogenic ROM restrictions were $15 \pm 6,44 \pm 5$ and $36 \pm 8^{\circ}$ in the control, Im, and Im + CBX groups, respectively (Fig. 3a). The myogenic ROM restrictions were significantly larger in both $\mathrm{Im}$ and $\mathrm{Im}+\mathrm{CBX}$ groups compared to the control $(P<0.0005, \mathrm{~F}=60.4$, $\eta_{\mathrm{P}}^{2}=0.81$, one-way ANOVA; both $P<0.05$, Tukey's post hoc test), and myogenic ROM restriction in the Im + CBX group was smaller than in the Im group $(P<0.05$, Tukey's post hoc test). Arthrogenic ROM restrictions were $0 \pm 8,30 \pm 5$, and $27 \pm 8^{\circ}$ in the control, Im, and Im $+\mathrm{CBX}$ groups, respectively (Fig. 3b). Arthrogenic ROM restrictions in the Im 


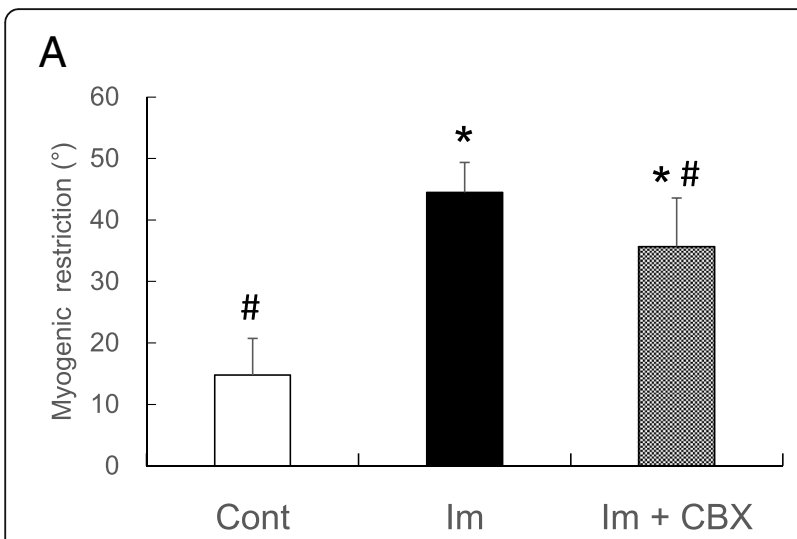

B

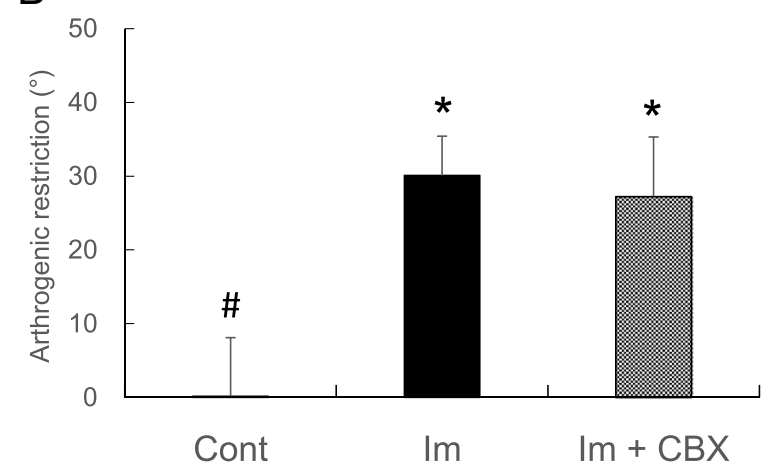

Fig. 3 Histograms showing changes in knee extension ROM restriction. a Myogenic restrictions. Celecoxib treatment partially reduced myogenic contracture induced by joint immobilization. b Arthrogenic restriction. Celecoxib treatment did not reduce arthrogenic contracture induced by joint immobilization. ${ }^{*} P<0.05$ vs. control group; ${ }^{\#} P<0.05$ vs. Im group. Data are expressed as means \pm SD $(n=10)$

and $\mathrm{Im}+\mathrm{CBX}$ groups were also significantly larger compared to the control $\left(P<0.0005, \mathrm{~F}=54.3, \eta_{\mathrm{P}}^{2}=0.80\right.$ for the ANOVA; both $P<0.05$ for the Tukey's post hoc test). There was no difference in arthrogenic ROM restriction between the Im and Im + CBX groups.

\section{Muscle length, sarcomere length, and serial number}

The semitendinosus muscle length, sarcomere length, and serial number are shown in Table 1 . Muscle length on the experimental side decreased in both the Im and Im + CBX groups compared to each contralateral side and the control $(P<0.05)$. The muscle length ratios (experimental side/contralateral side) of the two immobilized groups were significantly decreased compared to the control. Between the two immobilized groups, the muscle length ratio of the $\mathrm{Im}+\mathrm{CBX}$ group was larger than the Im group $(P<0.05$, Fig. 4a). Changes in sarcomere number were similar to those in muscle length ratio (Fig. 4b), but there were no differences in sarcomere length among the three groups (Table 1).

\section{Joint histology and histomorphometry}

In the semitendinosus muscles, muscle fibers were orderly arranged and apparent signs of inflammation were hardly observable in all three groups.

Representative images of the posterior capsule of the knee joint in HE sections are shown in Fig. 5a-f. In the control group, the joint capsule was thin and densely stained. Infiltrated cells were scarcely seen throughout the knee joint tissue (Fig. 5a, d). In AFMG sections, posterior joint capsules were densely stained green, indicating collagen fibers. In both Im and Im + CBX groups, the posterior capsules were thickened with edema and stained pale-green in AFMG sections. Fibrotic changes characterized by increased collagen deposition was not apparent in both Im and Im + CBX groups. Blood-derived cells (mainly red blood cells) had increased, especially in postero-superior portions near the synovial-cartilage junction in the knee joint cavities. Inflammatory infiltrates were rarely seen in posterior joint capsules (Table 2, Fig. 5b, c, e, f). These pathological changes were approximately comparable between $\mathrm{Im}$ and $\mathrm{Im}+\mathrm{CBX}$ groups. Mean hemorrhage scores showed a significant increase in both Im and Im + CBX groups compared to the control

Table 1 Semitendinosus muscle length, sarcomere length, and serial sarcomere number

\begin{tabular}{|c|c|c|c|c|c|c|c|}
\hline \multirow[t]{2}{*}{ Group } & & \multicolumn{2}{|l|}{ Muscle } & \multicolumn{4}{|l|}{ Sarcomere } \\
\hline & & Length (mm) & Rt/Lt ratio (\%) & Length $(\mu \mathrm{m})$ & Rt/Lt ratio (\%) & Number & $\overline{\text { Rt/Lt ratio (\%) }}$ \\
\hline \multirow[t]{2}{*}{ Control } & $\mathrm{Rt}$ & $51.1 \pm 1.3$ & $101 \pm 3$ & $2.7 \pm 0.2$ & $101 \pm 3$ & $20016 \pm 691$ & $99 \pm 2$ \\
\hline & $\mathrm{Lt}$ & $50.8 \pm 1.5$ & & $2.7 \pm 0.2$ & & $20165 \pm 625$ & \\
\hline \multirow[t]{2}{*}{ Im } & Rt & $46.4 \pm 2.1^{*}$ & $92 \pm 5^{\dagger}$ & $2.7 \pm 0.1$ & $98 \pm 4$ & $16892 \pm 316^{*}$ & $92 \pm 4^{\dagger}$ \\
\hline & $\mathrm{Lt}$ & $50.8 \pm 0.6$ & & $2.8 \pm 0.1$ & & $18047 \pm 505$ & \\
\hline \multirow[t]{2}{*}{$I m+C B X$} & $\mathrm{Rt}$ & $46.6 \pm 0.9^{*}$ & $97 \pm 3^{\dagger \S}$ & $2.8 \pm 0.1$ & $98 \pm 4$ & $16933 \pm 316^{*}$ & $97 \pm 3^{+\S}$ \\
\hline & Lt & $49.1 \pm 0.8$ & & $2.8 \pm 0.1$ & & $17291 \pm 155$ & \\
\hline
\end{tabular}

Values are shown as means \pm SD

*indicates significant difference from contralateral $(\mathrm{Lt})$ side $(P<0.05)$

${ }^{+}$indicates significant difference from Control $(P<0.05)$

$\varsigma_{\text {indicates significant difference from } \operatorname{Im}(P<0.05)}$ 


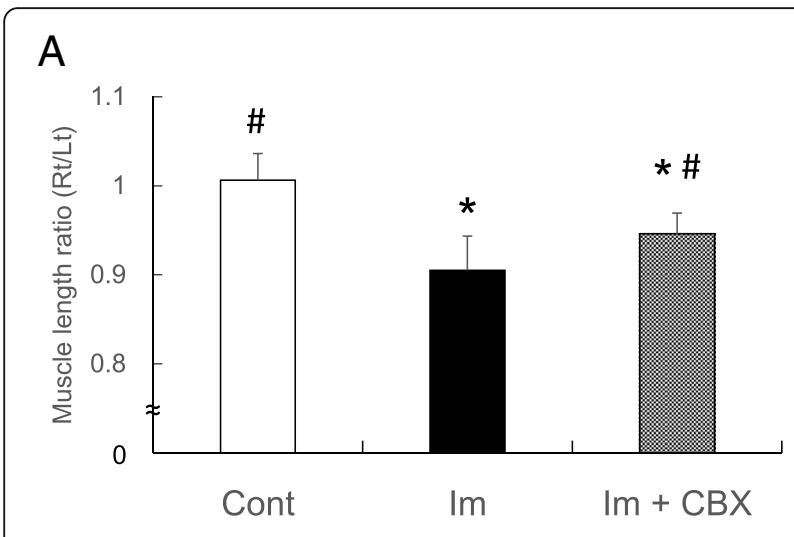

B

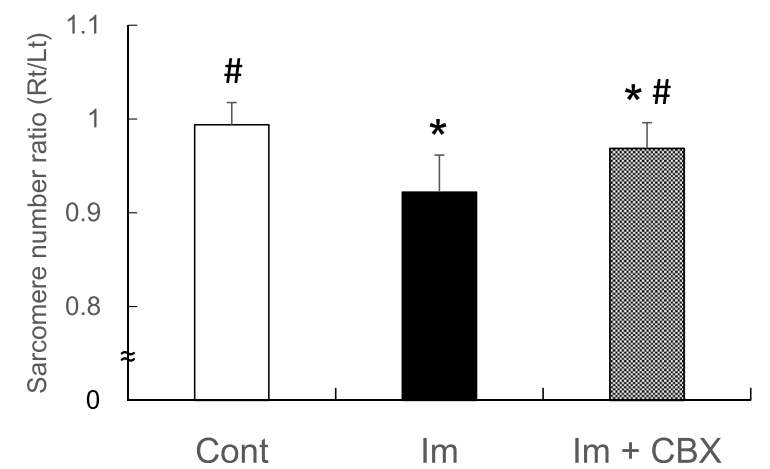

Fig. 4 Histograms showing morphological changes in the semitendinosus muscles. a Muscle length ratio (experimental side (Rt)/contralateral side (Lt)). b Sarcomere number ratio (experimental side (Rt)/contralateral side $(\mathrm{Lt})$ ). Decrease in both muscle length ratio and sarcomere number ratio were partially prevented by celecoxib treatment. ${ }^{*} P<0.05$ vs. control group; ${ }^{\#} P<0.05$ vs. Im group. Data are expressed as means \pm SD $(n=10)$

$(P<0.05)$, but there was no difference between the Im and Im + CBX groups (Fig. 5g).

The postero-superior synovial lengths were $6.2 \pm$ $1.0 \mathrm{~mm}, 3.8 \pm 0.5 \mathrm{~mm}(P<0.05)$, and $3.61 \pm 0.3 \mathrm{~mm}$ $(P<0.05)$ in the control, Im, and Im + CBX groups, respectively. The postero-inferior synovial lengths were $1.6 \pm 0.2 \mathrm{~mm}, 0.8 \pm 0.2 \mathrm{~mm}(P<0.05)$, and $0.8 \pm$ $0.3 \mathrm{~mm}(P<0.05)$, and the total lengths of the posterior synovial membrane were $7.7 \pm 1.1 \mathrm{~mm}, 4.6 \pm$ $0.5 \mathrm{~mm}(P<0.05)$, and $4.4 \pm 0.3 \mathrm{~mm}(P<0.05)$ in the control, Im, and Im + CBX groups, respectively. The posterior synovial membranes in the $\mathrm{Im}$ and $\mathrm{Im}+$ CBX groups were significantly shorter compared to the control (Fig. 5h). However, there was no difference between the two immobilized groups in any of the synovial length parameters.

\section{Discussion}

The key finding of the present study is that the COX-2 inhibitor celecoxib can partially prevent myogenic contracture by acting on knee flexor muscles following joint immobilization. However, arthrogenic contracture could not be inhibited by celecoxib treatment due to changes in articular components. These results indicate that the COX-2 mediated pathway, which is involved in inflammation and nociception, contributes to the formation of myogenic contracture independently of joint immobilization. This study is therefore the first to provide the beneficial effects of NSAID on the prevention of immobilizationinduced joint contracture.

The passive extensibility of skeletal muscle is determined by several anatomic and physiologic properties, with maximal muscle length being one important determinant of joint ROM [1, 29]. In this study, we demonstrated that the length of semitendinosus muscles in immobilized knee joints shortened resulting from a reduction in sarcomere number. This is in accordance with previous reports [29]. These changes were attenuated by celecoxib treatment concomitantly with myogenic ROM restriction. These results suggest that the COX-2 mediated pathway is involved in the regulation of muscle length by changing sarcomere number in the immobilized joint. The COX-2 also regulates skeletal muscle regeneration [30, 31], hypertrophy [32], and recovery from atrophy [33]. Passive extensibility is influenced by muscle size [29]. However, the effects of COX2 inhibitor on muscle atrophy following immobilization is unknown. Further study on muscle atrophy may elucidate the mechanisms behind CBX-mediated attenuation of muscular contracture.

In guinea pigs, muscle contraction in a shortened position accelerates the reduction in extensibility with sarcomere loss [34]. Huet de la Tour et al. [35] also reported that spasm of soleus muscles elicit a decrease in passive extensibility and a marked reduction of sarcomere number in guinea pigs. These results suggests that muscle contraction in a shortened position is key in the determination of muscle length. In addition, knee joint nociception also induces involuntary muscle contraction in biceps femoris muscles [36]. Several reports indicate that joint immobilization induces inflammatory conditions within affected joints, such as an increase in joint breadth [19], synovial cell proliferation, and mononuclear infiltration [13], as well as an upregulation of inflammatory cytokines [14]. In addition, we found pathological changes such as blood cell infiltration into intracapsular spaces and joint capsule edema in Im and Im + CBX groups. Interestingly, it has been reported that COX-1 and COX-2 are upregulated in synoviocytes and chondrocytes in immobilized rat knee joints [37]. Celecoxib inhibits prostaglandin $\mathrm{E}_{2}$ synthase, mediated by COX-2, and relieves pain [23]. Taken together, successful celecoxib treatment for alleviating myogenic contracture may be due to inhibition of the reduction in sarcomere number and muscle length via inhibition of 


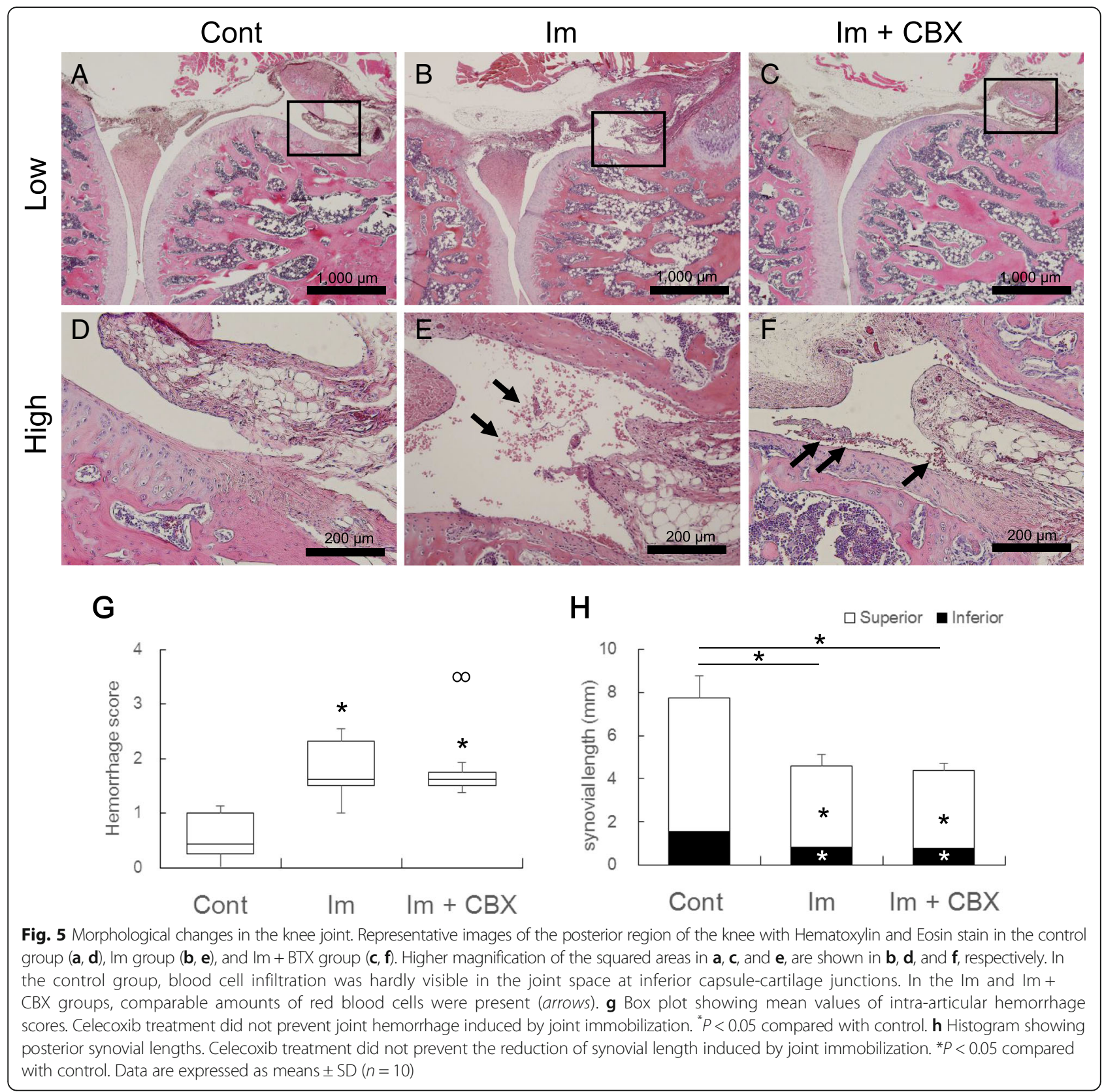

Table 2 Hemorrhage scores in each part of the knee joint cavity

\begin{tabular}{lllllll}
\hline Group & Antero- & & & Postero- & & Average \\
\cline { 2 - 3 } & Superior & Inferior & & Superior & Inferior & \\
\hline Control & $0.5 \pm 0.5$ & $0.5 \pm 0.5$ & & $0.6 \pm 0.5$ & $0.5 \pm 0.5$ & $0.5 \pm 0.4$ \\
Im & $1.8 \pm 0.8^{\dagger}$ & $1.6 \pm 0.5^{\dagger}$ & & $2.3 \pm 1.1^{\dagger}$ & $1.4 \pm 1.0^{\dagger}$ & $1.8 \pm 0.6^{\dagger}$ \\
Im + CBX & $1.5 \pm 0.8^{\dagger}$ & $1.3 \pm 0.8^{\dagger}$ & & $2.0 \pm 0.8^{\dagger}$ & $1.7 \pm 1.1^{\dagger}$ & $1.8 \pm 0.6^{\dagger}$ \\
\hline
\end{tabular}

Values are shown as means \pm SD

${ }^{+}$indicates significant difference from Control $(P<0.05)$ the COX-2-mediated inflammatory nociception following joint immobilization.

In addition, qualitative and quantitative changes in connective tissue are believed to be a leading candidate as the cause of myogenic contracture $[1,29]$. Endo- and perimysial connective tissue have been shown to increase in the rat soleus muscle when immobilized for 3 weeks [38, 39]. Perimysial collagen fibril arrangement also alters in immobilized rat soleus muscles [38] with a concomitant reduction in joint ROM [3]. In the present study, the sarcomere length at a stretched position, which is theoretically inhibited by the connective tissue surrounding the muscle fibers and 
bundles, was unaltered by joint immobilization. However, this result does not necessarily deny the involvement of intramuscular connective tissue in muscle extensibility. The semitendinosus muscles were pulled by a weight of $16 \mathrm{~g}$, and a stronger or weaker traction was not tested. In addition, changes in mechanical properties may occur in non-semitendinosus muscle, or in sliding between adjacent muscles. Thus, further study is needed to determine the full effects of celecoxib on the volume and mechanical properties of intramuscular collagen.

Celecoxib had no effect on arthrogenic contracture, unlike myogenic contracture. Trudel et al. [4] noted that structural reorganization in the joint posterior capsule (shortening due to adhesion between folds, type I collagen deposition, and advanced glycation end products) is responsible for arthrogenic joint contracture following long-lasting ( $>4$ weeks) joint immobilization in flexion [4]. In this study, the lengths of the posterior capsules were comparably shortened in both Im and Im + CBX groups. Celecoxib had no effect on arthrogenic contracture formation, which may be due to a failure to inhibit the shortening of the joint capsule. This result also suggests that the joint capsule length may be shortened by joint immobilization regardless of the inflammation.

Several studies report that the prevention of arthrogenic contracture is achieved by intraarticular injection of decorin, which inhibits the activity of transforming growth factor-beta [40], hyaluronic acid [21, 22], mytomycin C [41], and celecoxib [42]. These previous reports used animal models of traumatic contracture or a combination of joint trauma and immobilization, both of which show prominent inflammatory changes in affected joints, unlike in an immobilized model. In an immobilized model, arthrogenic contracture is alleviated by intraarticular injection of corticosteroid triamcinolone in rats [43]. However, as an adverse effect, triamcinolone-treated rat joints showed a disruption with a significantly lighter weight load than the untreated control. A recent study showed that intra-articular injection of hyaluronan could prevent fibrotic and inflammatory changes in immobilized joint capsules [21]. Exogenous hyaluronan treatment stimulates hyaluronan synthesis and reduces pro-inflammatory mediators in osteoarthritic joints [44]. The reason for this discrepancy between celecoxib and hyaluronan remain unexplained. Actions except anti-inflammation of hyaluronan, e.g., hydrodynamic changes, might be in part due to inhibit arthrogenic contracture.

Interestingly, microscopic observation revealed that intra-articular hemorrhages increased comparably in both Im and Im + CBX groups compared to the control. To test the possibility of developing joint damage caused by the mechanical stress during ROM measurement, we performed histological observation in three-week-immobilized knee joints without ROM measurement, additively $(n=2)$. The observation confirmed that joint immobilization itself contributes to bleeding. Coagulating blood not only induces joint damage [45] but also accelerates immobilizationinduced synovial adhesions and capsular shortening in rats [28]. The mechanism of joint hemorrhage induced by joint immobilization is unknown. However, if it is possible to prevent joint hemorrhage during immobilization, arthrogenic joint contracture might be alleviated.

Generally, passive movement is used as a strategy for the treatment of joint contracture. However, joint movement cannot be done in acute post-surgery phases or for orthopedic diseases such as joint fractures, muscle, and ligament injuries. In addition, it is not clear whether these interventions are clinically effective on joint mobility $[9,10]$. An important finding of this study is that even immobilization-induced contracture can be attenuated by oral administration of celecoxib, which is advantageous because it can be provided during periods of immobilization. This suggests that anti-inflammatory drug celecoxib is a possible option for the prevention of joint contracture followed by immobilization.

There are several limitations to the study. First, we observed the effects of joint immobilization and celecoxib treatment on joint contracture formation at only one time point. However, changes in the degree of contribution of both articular and muscle components in joint contracture formation depend on the period of immobilization [4]. Second, we measured the ROMs with only a single load, not an escalating load which provide a more detailed analysis of immobility. Third, administration of celecoxib in this study (50 mg/kg dose) is approximately $10 \mathrm{X}$ higher than that used by humans. In addition, the half-life for celecoxib in male rats is significantly shorter than that in human $(3.7 \mathrm{~h}$ vs $11 \mathrm{~h})$ [46]. Fourth, COX-2 activity was not measured to confirm drug efficacy and we did not investigate the roles of COX-2 pathways on the formation of joint contracture. In addition, we have not directly shown the anti-inflammatory effects of Celecoxib in immobilized joints. Therefore, whether this celecoxib efficacy could be extended for clinical use of joint contracture still requires further studies.

\section{Conclusions}

The present study revealed that the oral administration of COX-2 inhibitor celecoxib partially reduced myogenic ROM restriction concomitantly with knee flexor muscle shortening following immobilization. This result implies that inflammation and nociception are involved in myogenic contracture formation independently of joint immobilization. Celecoxib had a significant efficacy for prevention of joint contracture following immobilization in a rat model. 


\section{Abbreviations}

CBX: Celecoxib; COX: Cyclooxygenase; HE: Hematoxylin and eosin; Im: Immobilization; NSAID: Non-steroidal anti-inflammatory drug; ROM: Range of motion

\section{Acknowledgements}

Special thanks to Seiichi Kawamata and Tomoyuki Kurose (Hiroshima University) for their advice and expertise. We also acknowledge the technical assistance of Kengo Minamimoto, Nodoka Eguchi, Shunsuke Wakisaka, Yuta Sakamoto, Shogo Takagi, Koji Takagaki, Akie Koshi, and Haruna Motokawa (Hiroshima International University).

\section{Funding}

Financial support of this study was provided by the Department of Rehabilitation, Faculty of Rehabilitation, Hiroshima International University and a Grant-in-Aid for Scientific Research from the Ministry of Education, Culture, Sport, Science, and Technology of Japan \#22700564.

\section{Availability of data and materials}

All the data supporting our findings is contained within the manuscript.

\section{Authors' contributions}

$\mathrm{JO}$ contributed to the study design and the analysis and interpretation of data. JO, AK, and RT contributed to the acquisition of data. JO, NK, and HM contributed to the manuscript preparation. All authors read and approved the final manuscript.

\section{Competing interest}

The authors declare that they have no competing interest.

\section{Consent for publication}

Not applicable.

\section{Ethic approval}

This study was carried out upon receiving approval by the Committee of Research Facilities for Laboratory Animal Sciences at Hiroshima International University (permission number: AE10-010).

\section{Author details}

${ }^{1}$ Department of Rehabilitation, Faculty of Rehabilitation, Hiroshima International University, Kurose- Gakuendai 555-36, Higashi-Hiroshima, Hiroshima 739-2695, Japan. ${ }^{2}$ Graduate School of Medical Technology and Health Welfare Sciences, Hiroshima International University, Higashi-Hiroshima, Hiroshima, Japan. ${ }^{3}$ Department of Rehabilitation Science, Graduate School of Health Sciences, Kobe University, Tomogaoka 7-10-2, Suma-ku, Kobe, Hyogo, Japan.

\section{Received: 10 December 2015 Accepted: 17 October 2016} Published online: 24 October 2016

\section{References}

1. Farmer SE, James M. Contractures in orthopaedic and neurological conditions: a review of causes and treatment. Disabil Rehabil. 2001;23(13): 549-58.

2. Okita M, Nakano J, Kataoka H, Sakamoto J, Origuchi T, Yoshimura T. Effects of therapeutic ultrasound on joint mobility and collagen fibril arrangement in the endomysium of immobilized rat soleus muscle. Ultrasound Med Biol. 2009;35(2):237-44

3. Okita M, Yoshimura T, Nakano J, Motomura M, Eguchi K. Effects of reduced joint mobility on sarcomere length, collagen fibril arrangement in the endomysium, and hyaluronan in rat soleus muscle. J Muscle Res Cell Motil. 2004;25(2):159-66.

4. Trudel G, Laneuville O, Coletta E, Goudreau L, Uhthoff HK. Quantitative and temporal differential recovery of articular and muscular limitations of knee joint contractures; results in a rat model. J Appl Physiol. 2014;117(7):730-7.

5. Usuba M, Akai M, Shirasaki Y, Miyakawa S. Experimental joint contracture correction with low torque-long duration repeated stretching. Clin Orthop Relat Res. 2007:456:70-8

6. Usuba M, Miyanaga Y, Miyakawa S, Maeshima T, Shirasaki Y. Effect of heat in increasing the range of knee motion after the development of a joint contracture: an experiment with an animal model. Arch Phys Med Rehabil. 2006:87(2):247-53.

7. Hildebrand KA, Zhang M, Germscheid NM, Wang C, Hart DA. Cellular, matrix and growth factor components of the joint capsule are modified early in the process of posttraumatic contracture formation in a rabbit model. Acta Orthop. 2008;79(1):116-25.

8. Furia JP, Willis FB, Shanmugam R, Curran SA. Systematic review of contracture reduction in the lower extremity with dynamic splinting. Adv Ther. 2013;30(8):763-70.

9. Prabhu RK, Swaminathan N, Harvey LA. Passive movements for the treatment and prevention of contractures. Cochrane Database Syst Rev. 2013;12:CD009331.

10. Katalinic OM, Harvey LA, Herbert RD. Effectiveness of stretch for the treatment and prevention of contractures in people with neurological conditions: a systematic review. Phys Ther. 2011:91(1):11-24.

11. Kaneguchi A, Ozawa J, Kawamata S, Kurose T, Yamaoka K. Intermittent wholebody vibration attenuates a reduction in the number of the capillaries in unloaded rat skeletal muscle. BMC Musculoskelet Disord. 2014;15:315.

12. Kaneguchi A, Ozawa J, Moriyama H, Yamaoka K. Structures responsible for the formation of knee joint contracture secondary to adjuvant-induced arthritis in a rat model. Iryoukougaku Zasshi. 2015;9:1-12.

13. Michelsson JE, Hunneyball IM. Inflammatory involvement in rabbit knee following immobilization and resulting in osteoarthritis. Scand J Rheumatol. 1984;13(3):273-81.

14. Yabe $Y$, Hagiwara $Y$, Suda H, Ando A, Onoda $Y$, Tsuchiya M, Hatori K, Itoi E. Joint immobilization induced hypoxic and inflammatory conditions in rat knee joints. Connect Tissue Res. 2013;54(3):210-7.

15. Hashmi JA, Yashpal K, Holdsworth DW, Henry JL. Sensory and vascular changes in a rat monoarthritis model: prophylactic and therapeutic effects of meloxicam. Inflamm Res. 2010:59(8):667-78.

16. Kaur S, Bijjem KR, Sharma PL. Anti-inflammatory and antihyperalgesic effects of the combination of ibuprofen and hemin in adjuvant-induced arthritis in the Wistar rat. Inflammopharmacology. 2011;19(5):265-72.

17. Laird JM, Herrero JF, Garcia de la Rubia P, Cervero F. Analgesic activity of the novel COX-2 preferring NSAID, meloxicam in mono-arthritic rats: central and peripheral components. Inflamm Res. 1997:46(6):203-10.

18. Michelsson JE, Juntunen S, Valtakari T. Methylprednisolone has a preventive effect on the development of radiological changes, thickening and stiffening of the rabbit knee following immobilization. Clin Orthop Relat Res. 1990;8(5):439-43.

19. Michelsson JE. The effect of ibuprofen on the thickening, stiffening and development of degenerative changes in the rabbit knee following immobilization. Scand J Rheumatol. 1980;9(3):141-4.

20. Trudel G, Uhthoff HK. Contractures secondary to immobility: is the restriction articular or muscular? An experimental longitudinal study in the rat knee. Arch Phys Med Rehabil. 2000;81(1):6-13.

21. Kanazawa K, Hagiwara Y, Tsuchiya M, Yabe Y, Sonofuchi K, Koide M, Sekiguchi T, Itaya N, Ando A, Saijo Y, et al. Preventing effects of joint contracture by high molecular weight hyaluronan injections in a rat immobilized knee model. Int J Clin Exp Pathol. 2015;8(4):3426-40.

22. Wang M, Liu C, Xiao W. Intra-articular injection of hyaluronic acid for the reduction in joint adhesion formation in a rabbit model of knee injury. Knee Surg Sports Traumatol Arthrosc. 2014;22(7):1536-40.

23. Zweers MC, de Boer TN, van Roon J, Bijlsma JW, Lafeber FP, Mastbergen SC. Celecoxib: considerations regarding its potential disease-modifying properties in osteoarthritis. Arthritis Res Ther. 2011:13(5):239.

24. Nagai M, Aoyama T, Ito A, lijima H, Yamaguchi S, Tajino J, Zhang X, Akiyama $\mathrm{H}$, Kuroki $\mathrm{H}$. Contributions of biarticular myogenic components to the limitation of the range of motion after immobilization of rat knee joint. BMC Musculoskelet Disord. 2014;15:224.

25. Moriyama H, Tobimatsu Y, Ozawa J, Kito N, Tanaka R. Amount of torque and duration of stretching affects correction of knee contracture in a rat model of spinal cord injury. Clin Orthop Relat Res. 2013:471(11):3626-36.

26. Ando A, Suda H, Hagiwara $Y$, Onoda $Y$, Chimoto E, Itoi E. Remobilization does not restore immobilization-induced adhesion of capsule and restricted joint motion in rat knee joints. Tohoku J Exp Med. 2012;227(1):13-22.

27. Moriyama H, Yoshimura O, Kawamata S, Takemoto H, Saka Y, Tobimatsu Y. Alteration of knee joint connective tissues during contracture formation in spastic rats after an experimentally induced spinal cord injury. Connect Tissue Res. 2007;48(4):180-7.

28. Onoda $Y$, Hagiwara $Y$, Ando A, Watanabe $T$, Chimoto $E$, Suda $H$, Yabe $Y$, Saijo $Y$, Itoi E. Joint haemorrhage partly accelerated immobilization-induced 
synovial adhesions and capsular shortening in rats. Knee Surg Sports Traumatol Arthrosc. 2014;22(11):2874-83.

29. Gajdosik RL. Passive extensibility of skeletal muscle: review of the literature with clinical implications. Clin Biomech. 2001;16(2):87-101.

30. Bondesen BA, Mills ST, Kegley KM, Pavlath GK. The COX-2 pathway is essential during early stages of skeletal muscle regeneration. Am J Physiol Cell Physiol. 2004;287(2):C475-83.

31. Shen W, Prisk V, Li Y, Foster W, Huard J. Inhibited skeletal muscle healing in cyclooxygenase-2 gene-deficient mice: the role of PGE2 and PGF2alpha. J Appl Physiol. 2006;101(4):1215-21.

32. Novak ML, Billich W, Smith SM, Sukhija KB, McLoughlin TJ, Hornberger TA, Koh TJ. COX-2 inhibitor reduces skeletal muscle hypertrophy in mice. Am J Physiol Regul Integr Comp Physiol. 2009;296(4):R1132-9.

33. Bondesen BA, Mills ST, Pavlath GK. The COX-2 pathway regulates growth of atrophied muscle via multiple mechanisms. Am J Physiol Cell Physiol. 2006; 290(6):C1651-9.

34. Tabary JC, Tardieu C, Tardieu G, Tabary C. Experimental rapid sarcomere loss with concomitant hypoextensibility. Muscle Nerve. 1981;4(3):198-203.

35. Huet de la Tour E, Tardieu C, Tabary JC, Tabary C. Decrease of muscle extensibility and reduction of sarcomere number in soleus muscle following a local injection of tetanus toxin. J Neurol Sci. 1979;40(2-3):123-31.

36. Yamashita I, Atsuta Y, Shimazaki S, Miyatsu M. [Effects of prostaglandin E2 and sodium hyaluronate on bradykinin induced knee joint pain in rat] Nihon Seikeigeka Gakkai Zasshi. 1995;69(9):735-43.

37. Trudel G, Desaulniers N, Uhthoff HK, Laneuville O. Different levels of COX-1 and COX-2 enzymes in synoviocytes and chondrocytes during joint contracture formation. J Rheumatol. 2001;28(9):2066-74.

38. Jarvinen TA, Jozsa L, Kannus $P$, Jarvinen $T L$, Jarvinen M. Organization and distribution of intramuscular connective tissue in normal and immobilized skeletal muscles. An immunohistochemical, polarization and scanning electron microscopic study. J Muscle Res Cell Motil. 2002;23(3):245-54.

39. Jozsa L, Thoring J, Jarvinen M, Kannus P, Lehto M, Kvist M. Quantitative alterations in intramuscular connective tissue following immobilization: an experimental study in the rat calf muscles. Exp Mol Pathol. 1988;49(2):267-78.

40. Fukui N, Fukuda A, Kojima K, Nakajima K, Oda H, Nakamura K. Suppression of fibrous adhesion by proteoglycan decorin. J Orthop Res. 2001;19(3):456-62.

41. Kocaoglu B, Akgun U, Nalbantoglu U, Poyanli O, Karahan M. Adhesion reduction after knee surgery in a rat model by mitomycin C. Knee Surg Sports Traumatol Arthrosc. 2011;19(1):94-8.

42. Li F, He B, Liu S, Fan C. Celecoxib effectively inhibits the formation of joint adhesions. Exp Ther Med. 2013;6(6):1507-11.

43. Clark DD, Weckesser EC. The influence of triamcinolone acetonide on joint stiffness in the rat. J Bone Joint Surg Am. 1971;53(7):1409-14.

44. Migliore A, Procopio S. Effectiveness and utility of hyaluronic acid in osteoarthritis. Clin Cases Miner Bone Metab. 2015;12(1):31-3.

45. van Meegeren ME, Roosendaal G, Barten-van Rijbroek AD, Schutgens RE, Lafeber FP, Mastbergen SC. Coagulation aggravates blood-induced joint damage in dogs. Arthritis Rheum. 2012;64(10):3231-9.

46. Paulson SK, Zhang JY, Breau AP, Hribar JD, Liu NW, Jessen SM, Lawal YM, Cogburn JN, Gresk CJ, Markos CS, et al. Pharmacokinetics, tissue distribution, metabolism, and excretion of celecoxib in rats. Drug Metab Dispos. 2000; 28(5):514-21.

\section{Submit your next manuscript to BioMed Central and we will help you at every step:}

- We accept pre-submission inquiries

- Our selector tool helps you to find the most relevant journal

- We provide round the clock customer support

- Convenient online submission

- Thorough peer review

- Inclusion in PubMed and all major indexing services

- Maximum visibility for your research

Submit your manuscript at www.biomedcentral.com/submit

) Biomed Central 